\title{
The Role of Lysyl Oxidase-like 2 in the Odontogenic Differentiation of Human Dental Pulp Stem Cells
}

\author{
Joo-Hyun Kim ${ }^{1,2,5}$, Eun-Hyang Lee ${ }^{3,5}$, Hye-jeong Park ${ }^{1}$, Eui-Kyun Park ${ }^{2}$, Tae-Geon Kwon ${ }^{4}$, Hong-In Shin ${ }^{2}$, \\ and Je-Yoel Cho ${ }^{1, *}$
}

\begin{abstract}
Adult human dental pulp stem cells (hDPSCs) are a unique population of precursor cells those are isolated from postnatal dental pulp and have the ability to differentiate into a variety of cell types utilized for the formation of a reparative dentin-like complex. Using LC-MS/MS proteomics approaches, we identified the proteins secreted from the differentiating hDPSCs in mineralization media. Lysyl oxidase-like 2 (LOXL2) was identified as a protein that was down-regulated in the hDPSCs that differentiate into odontoblast-like cells. The role of LOXL2 has not been studied in dental pulp stem cells. LOXL2 mRNA levels were reduced in differentiating hDPSCs, whereas the levels of other LOX family members including LOX, LOXL1, LOXL3, and LOXL4, are increased. The protein expression and secretion levels of LOXL2 were also decreased during odontogenic differentiation. Recombinant LOXL2 protein treatment to hDPSCs resulted in a dose-dependent decrease in the early differentiation and the mineralization accompanying with the lower levels of odontogenic markers such as DSPP, DMP-1 and ALP. These results suggest that LOXL2 has a negative effect on the differentiation of hDPSCs and blocking LOXL2 can promote the hDPSC differentiation to odontoblasts.
\end{abstract}

\section{INTRODUCTION}

Tooth regeneration is one of the ultimate goals of restoring the loss of natural teeth. Stem cell based strategies show promising potential for regenerating the entire tooth structure in rodents (Chai and Slavkin, 2003; Duailibi et al., 2004). Mesenchymal stem cells are multipotent progenitor cells that divide many times, and their progeny eventually give rise to skeletal tissues: cartilage, bone, tendon, ligament, marrow stroma, and dental tissues. Both intrinsic and extrinsic factors combine to control the molecular and cellular patterns of expression that result in specific tissues performing specific functions based on their molecular repertoire. Adult dental pulp tissues also contain a population of multipotent stem cells. Isolated hDPSCs that are expanded ex vivo would also be capable of regenerating a dentin/pulp-like structure in vivo under similar conditions (Gronthos et al., 2000). It was reported that hDPSCs can differentiate into odontoblasts, osteoblasts, adipocytes, chondrocytes, and active neurons (d'Aquino et al., 2007; Koyama et al., 2009; Yu et al., 2010). More specifically, it was shown that cells derived from dental pulp are capable of forming mineralized nodules in vitro in the presence of inductive media containing ascorbic acid, dexamethasone, and an excess of phosphate (Yokose et al., 2000).

The Lysyl oxidase-like 2 (LOXL2) is a member of the LOX family of proteins, which is composed of five paralogs. These are known as LOX, LOXL, LOXL2, LOXL3, and LOXL4 in humans (Kagan et al., 1995). Each LOX family protein contains a copper-binding motif, lysyl-tyrosyl-quinone (LTQ) residues, and a cytokine receptor-like (CRL) domain in its highly conserved carboxyl (C)-terminus (Hayashi et al., 2004a). The main molecular function of the LOX enzyme is lysyl oxidation to make protein cross-linking through lysine residues. Type I collagen is one of the well known targets of LOX proteins (Hayashi et al., 2004b). The catalytic domain contains conserved residues that are required for copper binding and formation of a lysyl-tyrosylquinone cofactor (Maki and Kivirikko, 2001). In contrast to the characteristic C-terminal domains, the LOX family members show sequence divergence in their amino $(\mathrm{N})$-terminal regions. In particular, LOXL2, LOXL3 and LOXL4 contain four scavenger receptor cysteine-rich (SRCR) domains in their N-terminal regions (Saito et al., 1997). The functional role of the SRCR domains in these three LOX paralogs has not yet been characterized; however, SRCR domains are known to be involved in the protein-protein interactions of several secreted or receptor proteins. While the regulation of gene expression of members of the LOX family has been well characterized in various cells and tissues (Rodriguez et al., 2008), the expressions and roles of LOX family in the human dental pulp stem cells have yet to be fully elucidated.

LOXL2 is expressed in many tissues with elevated levels in

\footnotetext{
${ }^{1}$ Department of Veterinary Biochemistry, Brain Korea 21 and Research Institute for Veterinary Science, College of Veterinary Medicine, Seoul National University, Seoul 151-742, Korea, ${ }^{2}$ Department of Pathology, School of Dentistry, Kyungpook National University, Daegu 700-412, Korea, ${ }^{3}$ ProtAnBio, Co., Seoul 151-742, Korea, ${ }^{4}$ Department of Oral and Maxillofacial Surgery, School of Dentistry, Kyungpook National University, Daegu 700-412, Korea, ${ }^{5}$ These authors contributed equally to this work.

*Correspondence: jeycho@snu.ac.kr
} 
reproductive tissues, such as placenta, uterus, and prostate (Jourdan-Le Saux et al., 1999). There are much similarities between odontogenic differentiation and osteogenic differentiation and, interestingly, LOXL2 has been found to be downregulated during BMP-induced osteogenic differentiation (Kaku et al., 2007). In this study, through LC-MS/MS-based proteomics approaches, we identified LOXL2 protein secreted at a significantly low level in early odontogenic differentiation of hDPSCs. We hypothesized that LOXL2 is down-regulated and has inhibitory role in the differentiation of hDPSCs. Since the role of LOXL2 in odontogenesis is not well elucidated, we investigated the role of LOXL2 in early odontogenic differentiation of hDPSCs.

\section{MATERIALS AND METHODS}

Human dental pulp stem cell isolation and culture Human adult third molar samples were extracted from the Department of Oral and Maxillofacial Surgery at Kyungpook National University Hospital. All of the protocols were reviewed by the IRB of Kyungpook National University Hospital, and patient consent forms were collected for each tooth sample. Sixteen patients were enrolled and one tooth was extracted from each patient. hDPSCs were isolated as previously described (Lee et al., 2010). Briefly, dental pulp tissues were isolated from the tooth and then digested in $3 \mathrm{mg} / \mathrm{ml}$ collagenase type I (Worthington, USA) for $1 \mathrm{~h}$ at $37^{\circ} \mathrm{C}$ in a humidified atmosphere containing $5 \% \mathrm{CO}_{2}$. After digestion, the cells were passed through a $70-\mu \mathrm{m}$ strainer (BD Falcon, USA) to obtain single-cell suspensions. The cells were seeded onto culture plates with MEM Alpha Modification medium ( $\alpha$-MEM; Hyclone, South Logan, UT) supplemented with $10 \%$ fetal bovine serum (Hyclone) and $1 \%$ antibiotic-antimycotic solution (GIBCO, USA) and then incubated at $37^{\circ} \mathrm{C}$ with $5 \% \mathrm{CO}_{2}$. The medium was replaced twice a week. Single colony was picked up the plated, expanded and the mesenchymal stem cells were confirmed by FACS analysis.

\section{Fluorescence-activated cell sorting (FACS)}

hDPSCs established from a single colony were characterized using fluorescence-activated cell sorting (FACS). A minimum of 10,000 cells were prepared in cold phosphate-buffered saline (PBS) containing $0.1 \%$ bovine serum albumin (BSA). Then, the cells were incubated for $1 \mathrm{~h}$ at $4^{\circ} \mathrm{C}$ in the dark with the following mouse anti-human monoclonal antibodies: fluorescein isothiocyanate (FITC)-labeled antibody against CD34, phycoerythrin (PE)-labeled antibodies against CD44 and CD73, and CD105 antibodies directly conjugated to FITC. After the incubation, cells were washed with $0.1 \%$ BSA in cold PBS and then analyzed using a FACSAria instrument (BD Bioscience, USA).

\section{Odontogenic differentiation}

For odontogenic differentiation, hDPSCs were seeded at $5 \times$ $10^{4}$ cells in 35-mm tissue culture plates and cultured until full confluence in $\alpha$-MEM. Then, the cells were incubated in $\alpha$ MEM supplemented with mineralization media that included 10 $\mathrm{mM} \beta$-glycerophosphate, $50 \mathrm{mM}$ ascorbic acid, and $0.1 \mathrm{mM}$ dexamethasone for odontogenic/osteogenic differentiation.

\section{Preparation of secreted proteins}

Secreted proteins were collected from hDPSCs. The cells were cultured until fully confluence in growth media. Then, cells were cultured in growth media or mineralization media. After 3 days, cells were washed by serum free media for 5 times and incubated with serum-free media for $24 \mathrm{~h}$. Proteins in conditioned media were precipitated with $10 \%(\mathrm{v} / \mathrm{v})$ trichloroacetic acid (TCA) (Sigma-Aldrich, USA) in $4^{\circ} \mathrm{C}$. Using centrifugation at $4 \times$ $10^{3} \mathrm{rpm}$ for $1 \mathrm{~h}$ at $4^{\circ} \mathrm{C}$ and ice-cold acetone washing for 5 times, secreted proteins were harvested. The protein pellets were air dried before use.

\section{Proteomics analysis by LC-MS/MS}

The secreted proteins were subjected in-gel trypsin digestion as previously reported (Narayanasamy et al., 2011). Mass spectrometry and data analysis were also performed as previously reported (Narayanasamy et al., 2011). Briefly, LCMS/MS analysis was done in Thermo Finnigan's ProteomeX workstation LTQ linear ion trap MS (Thermo Electron, USA) equipped with NSI sources (USA). After injection of the peptide samples to the mass spectrometry, data dependent acquisition mode $(\mathrm{m} / \mathrm{z} 300-1,800)$ was enabled, and each survey MS scan was followed by five MS/MS scans. The normalized collision energy was set at $35 \%$.

\section{RNA isolation and RT-PCR analysis}

RNA isolation and RT-PCR analysis were performed as previously described (Heo et al., 2011). Briefly, Total RNA was extracted from hDPSCs using TRIzol ${ }^{\circledR}$ (Invitrogen, USA) according to the manufacturer's protocol. Total RNA $(1 \mu \mathrm{g})$ was reverse-transcribed using the Omni Script Reverse Transcription Kit (QIAGEN, USA). The resulting cDNAs were used for the conventional RT-PCR or real-time quantitative RT-PCR. RT-PCR reactions were performed using GoTaq1 Flexi DNA polymerase (Promega, USA).

Real-time qRT-PCR analysis

Fluorescence-based real-time quantitative RT-PCR (qRT-PCR) was performed using a CFX Connect ${ }^{\top M}$ PCR detection system (Biorad, USA) with SYBR Green I dye (Molecular Probes, USA). For quantification, human glyceraldehyde-3-phosphate dehydrogenase (GAPDH) was used as a reference to normalize each sample. The primers used for the RT-PCR analysis are listed in Table 1.

\section{Western blot analysis}

Western blot analysis was performed as previously described (Lee et al., 2010). Briefly, hDPSCs were washed twice with cold PBS and lysed with RIPA buffer containing protease inhibitors (Roche, Germany). Total cell lysates were separated by SDS polyacrylamide gel electrophoresis and transferred to polyvinylidene fluoride (PVDF) membranes. For primary antibody labeling, rabbit anti-human LOXL2 polyclonal antibodies (1:2,000, GeneTex, USA) were used. HRP-conju-gated anti-rabbit IgG antibodies (1:2,000, Enzo ${ }^{\circledR}$ LifeScience, USA) were used as secondary antibodies. For a normalization control, anti- $\beta$-Actin monoclonal antibodies (1: 2,000, Sigma-Aldrich, USA) were used. Immunoreactive proteins were detected using Pierce ${ }^{\circledR}$ ECL Plus Substrate (Thermo Scientific, USA).

\section{Recombinant LOXL2 treatment}

hDPSCs were treated with recombinant human lysyl oxidaselike 2 (rhLOXL2, R\&D Systems, Inc., USA) protein during the mineralization and differentiation stages, at concentrations of $0.1,1$, or $10 \mathrm{ng} / \mathrm{ml}$ for 7 days. Cells were then stained with ALP or Alizarin Red S or lysed to collect total RNA or proteins for further analysis. 
Table 1. Primers used for RT-PCR analysis

\begin{tabular}{llc}
\hline Target gene & Primer sequence & Product size (bp) \\
\hline \multirow{2}{*}{ hLOX } & Forward: 5'-CAGAGGAGAGTGGCTGAAGG-3' & 223 \\
& Reverse: 5'-CCAGGTAGCTGGGGTTTACA-3' & 163 \\
hLOXL1 & Forward: 5'-CACCAGCATTACCACAGCAT-3' & \\
& Reverse: 5'-CCTGGGTATGAGAGGTGCAT-3' & 197 \\
hLOXL2 & Forward: 5'-GAGTTGCCTGCTCAGAAACC-3' & \\
& Reverse: 5'-GTTGTGGATCTGGGAGGAAA-3' & 232 \\
hLOXL3 & Forward: 5'-GTCCGGTGCAACCTACCTTA-3' & \\
hLOXL4 & Reverse: 5'-TCCCAGAGTCCCAGTACCAG-3' & 240 \\
hDSPP & Forward: 5'-GAGAGCTCCTTGGACCAGTG-3' & \\
& Reverse: 5'-GCCCTCATACTTCACCTCCA-3' & 280 \\
hDMP-1 & Forward: 5'-GAGGATAAAGGACAACATGG-3' & 130 \\
hALP & Reverse: 5'-AAGAAGCATCTCCTCGGC-3' & 162 \\
hGAPDH & Forward: 5'-GATCAGCATCCTGCTCATGTTC-3' & 200 \\
\hline
\end{tabular}

ALP and Alizarin Red S staining

Alkaline phosphatase (ALP) and Alizarin Red S staining were performed as previously described (Lee et al., 2010). Briefly, ALP activity was cytochemically assessed using an ALP staining kit according to the manufacturer's protocol (Sigma-Aldrich, USA). The mineralization of hDPSCs was evaluated by Alizarin Red S staining of cells in 24-well plates. The hDPSCs were grown in the mineralization media for 21 days, and then the cells were fixed with $4 \%$ paraformaldehyde (PFA) for $1 \mathrm{~h}$ at $4{ }^{\circ} \mathrm{C}$ and stained with Alizarin Red S (Sigma-Aldrich, USA).

Statistical analysis

Each real-time PCR experiment was done in triplicate. The significance of the differences was determined using two-tailed Student's $t$-test. All experiments were repeated at least three times independently. Results are represented as mean \pm SEM. A significant difference was considered for $\mathrm{P}<0.05$.

\section{RESULTS}

Isolation of hDPSCs from human third molars and characterization

Human dental pulp stem cells (hDPSCs) were isolated from extracted human teeth. The hDPSCs were able to form individual colonies in culture. Ten days after seeding, cells that formed single-colony clusters were identified (Fig. 1A). These cells showed a spindle-like morphology, which is generally observed in mesenchymal stem cells (MSCs). Colonies of hDPSCs were selected after evaluating the levels of the MSC-specific cell surface markers, CD44, CD73, and CD105, by FACS analysis. We used a hematopoietic surface molecule, CD34, as a negative control (Morsczeck et al., 2008). The isolated clones that tested positive for CD44, CD73, and CD105 and negative for CD34 were used for subsequent experiments (Fig. 1B). The differentiated cells from hDPSCs by mineralization media were positive for ALP staining, and the ALP mRNA expression levels were increased in hDPSCs during the early differentiation stage of hDPSCs with a maximum increase at day 5 (Fig. 1C).

Proteomic analysis of hDPSCs during early differentiation using LC-MS/MS

To identify the secreted proteins that are regulated during early odontogenic/osteogenic differentiation, hDPSCs were treated with mineralization media. Two sources of hDPSCs from two different individuals were used for the proteomics analysis. Three days after hDPSCs were treated with mineralization media, the conditioned media was collected and subjected to LCMS/MS analysis for the identification of differentially regulated secreted proteins. In this study, we identified and focused on the decreased secretory proteins of the hDPSC in mineralization media by selecting 1.5 -fold decrease of spectral counts in MM compared to GM (Supplementary Table 1). Among the down-regulated proteins, we selected LOXL2 for further studies, since the function of the LOXL2 has not been studied in the hDPSCs.

\section{Identification of LOXL2 and its expression during early} odontogenic differentiation

LOXL2 was chosen for further analysis, specifically to elucidate its role in the odontogenic differentiation of hDPSCs. We first tested the mRNA expression levels of selected candidates from 4 different hDPSC clones by qRT-PCR. As shown in Fig. 2A, LOXL2 mRNA levels were significantly decreased in all hDPSCs clones that were subjected to mineralization media for 3 days. When analyzed by Western blot, the protein levels of LOXL2 in the cell lysates were also decreased in the hDPSC in mineralization media (Fig. 2B). Secreted forms of LOXL2 were also dramatically decreased in conditioned media after exposure to mineralization media for 3 days (Fig. $3 \mathrm{C}$ ). This result confirmed the decreased levels of LOXL2, as determined by MS/MS analysis.

Interestingly, the LOXL2-positive band in the secretome as 
A

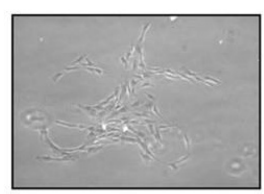

B
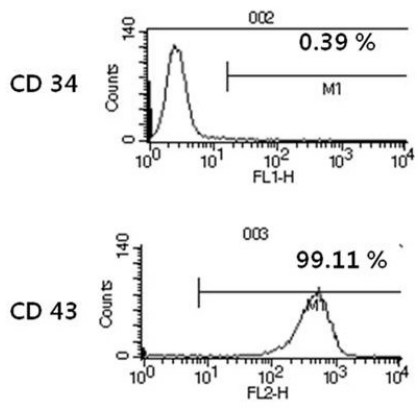

CD 105
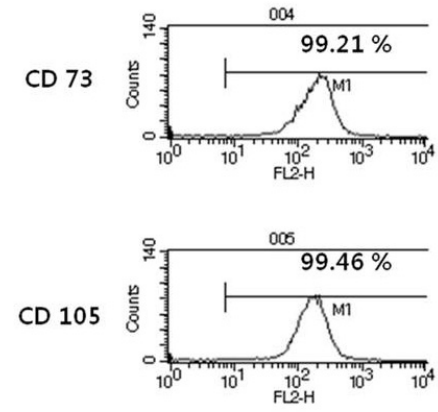

C

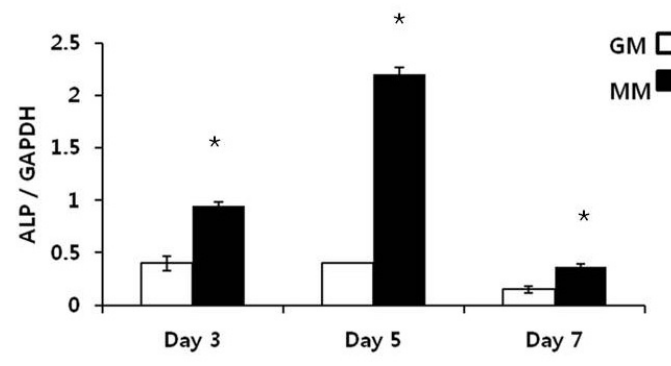

Fig. 1. Morphology and surface marker expression of hDPSCs. (A) The hDPSCs that grew from the dental pulp showed a fibroblast-like morphology. Magnification, 40x. (B) A representative results of FACS analysis of the cell surface protein markers CD44, CD73 and CD105 (MSC surface markers), and CD34 (hematopoietic surface protein) as a negative marker. (C) Confirmation of the ability of hDPSCs to differentiate into odontogenic/osteogenic cells via analysis of mRNA expression levels of alkaline phosphatase (ALP). ${ }^{*} \mathrm{P}<$ 0.05 versus control.
A

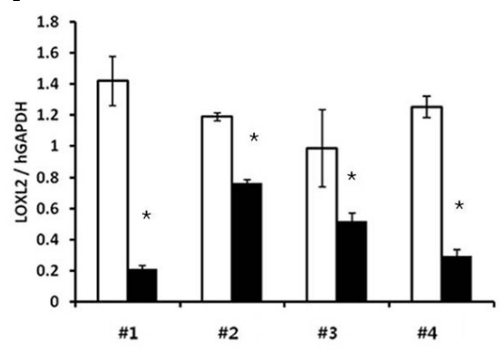

B

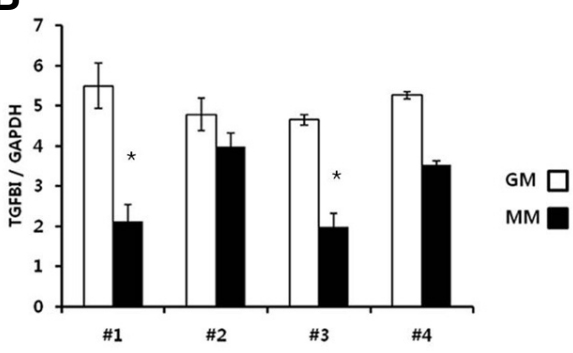

C

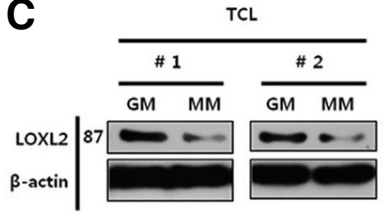

D

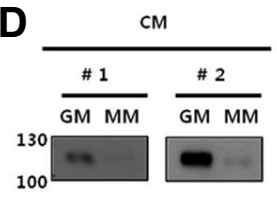

E

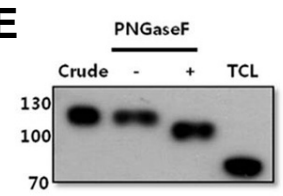

Fig. 2. Confirmation of LOXL2 expressions in early odontogenic differentiation. (A) The lysyl oxidase-like 2 (LOXL2) mRNA expression levels were confirmed by real-time qRT-PCR for the RNAs isolated from the hDPSCs exposed to either normal growth media (GM) or mineralization media (MM) for 3 days. $(B, C)$ LOXL2 expression levels in total cell lysates (TCL) (B) and in the secreted protein component from conditioned medium $(\mathrm{CM})(\mathrm{C})$, at the early odontogenic induction stage, were determined by Western blot analysis using an anti-LOXL2 antibody. (D) PNGase F treatment of secreted proteins resulted in a smaller size for LOXL2, indicating that its higher molecular weight is partially attributed to $\mathrm{N}$-glycosylation modification. ${ }^{*} P<0.05$ versus control. shown in Fig. $2 \mathrm{C}$ appeared to have a higher molecular weight (about $115 \mathrm{kDa}$ ) than that in the cell lysates (about $87 \mathrm{kDa}$ ) as shown in Fig. 2B. We hypothesized that the higher molecular weight in the conditioned media is possibly due to higher glycosylation of the secreted LOXL2 proteins. Thus, to confirm whether N-linked glycosylation occurs in secreted LOXL2, the proteins were treated with PNGase F (Fig. 2D). PNGase F, also known as $\mathrm{N}$-Glycosidase $\mathrm{F}$, is an amidase that cleaves between the innermost GlcNAc and asparagine residues of high mannose, hybrid, and complex oligosaccharides from $\mathrm{N}$-linked glycoproteins (Tretter et al., 1991). PNGase F treatment resulted in a reduction of the molecular weight of LOXL2 (about $100 \mathrm{kDa}$ ), suggesting that $\mathrm{N}$-linked glycosylation of LOXL2 proteins does occur. However, PNGase F cleavage of secreted LOXL2 did not result in the same molecular weight as the intracellular form of LOXL2. This finding suggests that there could be posttranslational modifications other than $\mathrm{N}$-glycosylation on the secreted LOXL2 form or that the intracellular LOXL2 form 
A

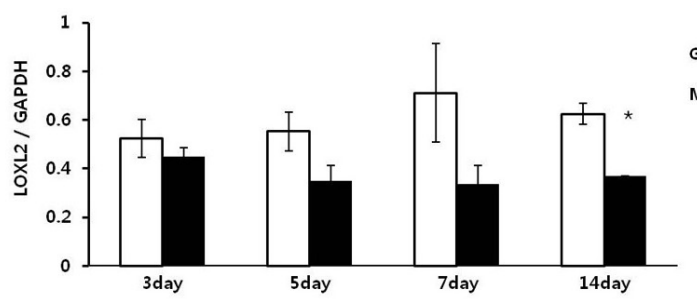

B

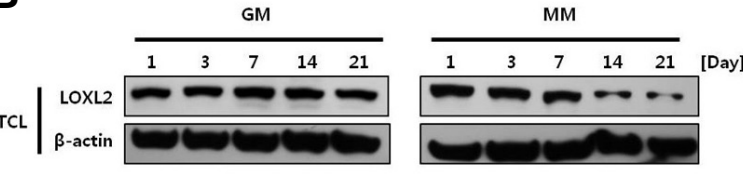

C

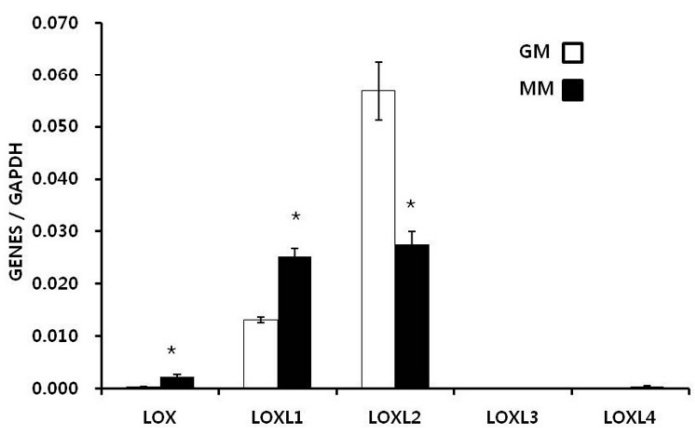

D

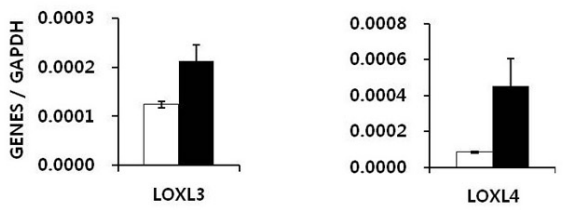

Fig. 3. The mRNA expression levels and protein levels of LOXL2 during odontogenic differentiation. (A) LOXL2 mRNA expression levels were monitored by qRT-PCR during odontogenic differentiation by mineralization media (MM). (B) The total cell lysates (TCL) of hDPSCs cultured in normal growth media (GM) or mineralization media (MM) for given days subjected to Western blot analysis. (C) The expression levels of LOX family members in the hDPSCs for 3 days of GM or MM were analyzed by real-time qRT-PCR. (D) Shown are magnified subsets of the LOXL3 and LOXL4 data from (C). ${ }^{*} \mathrm{P}<0.05$ versus control.

and the secreted form are different protein variants or isoforms. Further analysis is required to reveal the molecular identities of the intracellular and secreted LOXL2 forms.

\section{LOXL2 expression during odontogenic differentiation of hDPSCs}

LOXL2 expression patterns were analyzed during the differentiation process of hDPSCs into mineralizing cells by real-time qRT-PCR and Western blot. We confirmed that ALP is induced in hDPSCs during their early stage differentiation into mineralizing cells (Fig. 1C). LOXL2 mRNA expression levels were decreased from day 3 to day 14 in during the differentiation (Fig. $3 A)$. Furthermore, LOXL2 protein expression levels in total cell lysates of hDPSCs also decreased until day 21 of differentiation
A
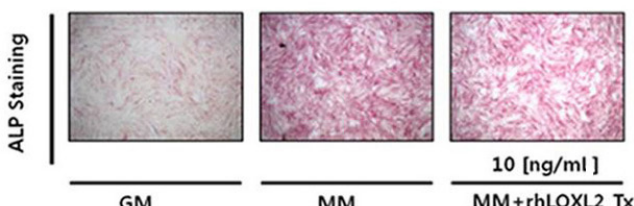

B
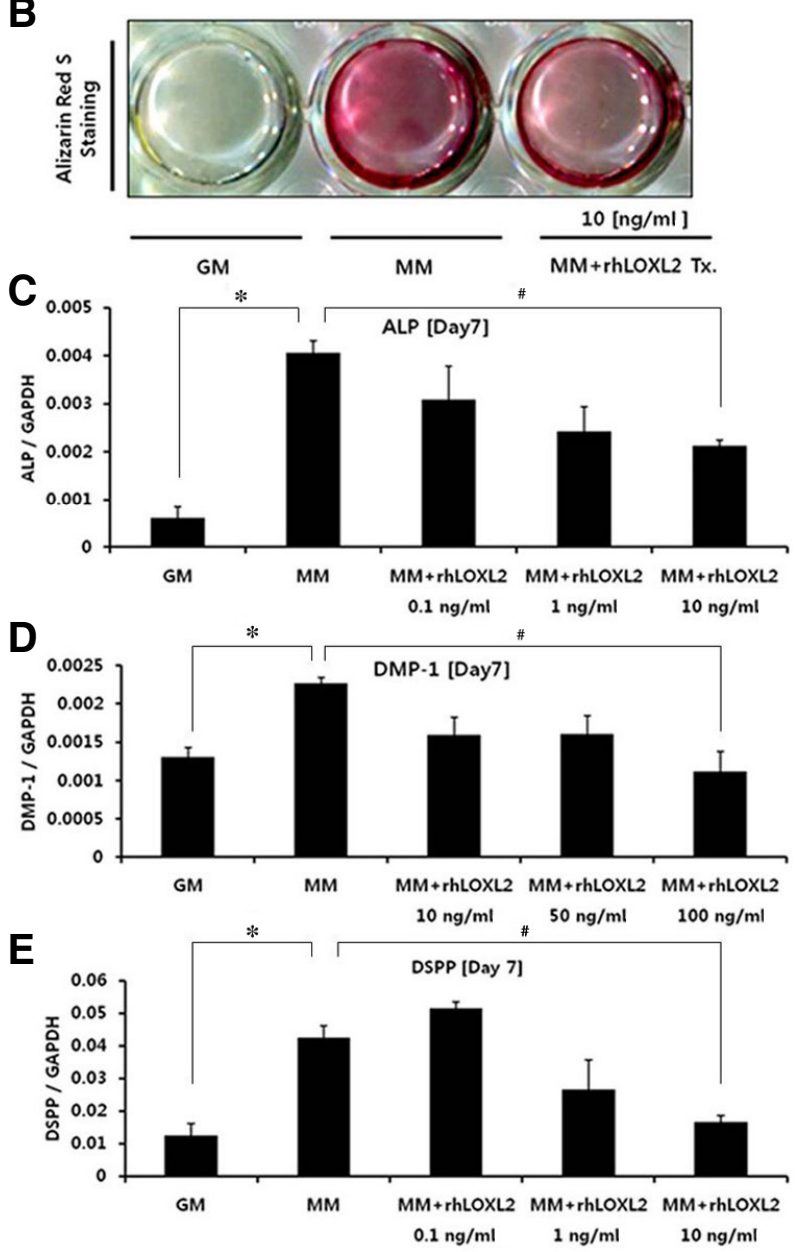

Fig. 4. The effect of rhLOXL2 treatment on hDPSCs. (A, B) hDPSCs were treated with normal growth media (GM) or mineralization media (MM) containing $10 \mathrm{ng} / \mathrm{ml}$ of rhLOXL2 for 7 days and then stained with ALP (A) and Alizarin Red S (B). (C) ALP expression levels were examined by qRT-PCR in hDPSC cells treated with rhLOXL2 at the concentrations of $0.1,1$, and $10 \mathrm{ng} / \mathrm{ml}$ in $\mathrm{MM}$ for 7 days. (D) The mRNA expression levels of DMP-1, an odontogenic-selective gene, were examined by qRT-PCR in the cells treated with rhLOXL2 at the concentrations of 10,50 , and 100 $\mathrm{ng} / \mathrm{mL}$ in MM for 7 days, (E) DSPP, another onodogenic selective gene, expression levels were also examined by qRT-PCR in hDPSCs treated with rhLOXL2 at concentrations of $1,10 \mathrm{ng} / \mathrm{ml}$ in MM for 7 days. *,$p<0.05$ versus control.

of hDPSCs (Fig. 3B). Our data suggest that LOXL2 mRNA and protein expression levels are decreased not only in early but also in late stages of hDPSCs differentiation into mineralizing cells.

Then, we examined the expression of other LOXL family 
Role of LOXL2 in the Odontogenic Differentiation of DPSC

Joo-Hyun Kim et al.

Table 2. Characteristics of the LOX family of proteins

\begin{tabular}{ccccc}
\hline LOX & Sequence length [aa] & Chromosome location & Subcellular location & Tissue specificity \\
\hline LOXL1 & 417 & Chr 5: 121.43-121.44 Mb & Secreted & $\begin{array}{c}\text { Heart, placenta, skeletal muscle, kidney, lung, } \\
\text { and pancreas } \\
\text { Ocular tissues (iris, ciliary body, lens, and } \\
\text { optic nerve); not detected in the retina }\end{array}$ \\
LOXL2 & 574 & Chr 15: 72.01-72.03 Mb & Secreted & $\begin{array}{c}\text { Seproductive tissues, placenta, uterus, and } \\
\text { prostate }\end{array}$ \\
LOXL3 & 774 & Chr 8: 23.21-23.28 Mb & Secreted & $\begin{array}{c}\text { Placenta, heart, ovary, testis, small intestine, } \\
\text { and spleen }\end{array}$ \\
LOXL4 & 753 & Chr 2: 74.61-74.63 Mb & Secreted & Skeletal muscle, testis, pancreas, and cartilage \\
\hline
\end{tabular}

members at the early stage of hDPSCs differentiation (day 3). Surprisingly, LOX and all other LOXL1 were increased at the early stage of differentiation (Fig. 3C). LOXL3 and LOXL4 expression levels were relatively low compared to others; however, these two were also increased in hDPSCs at the early state of differentiation (Fig. 3D).

\section{rhLOXL2 treatment of hDPSCs suppresses odontogenic differentiation}

To explore the role of LOXL2 in hDPSCs odontogenic differentiation, hDPSCs were treated with a recombinant human LOXL2 protein (rhLOXL2) together with mineralization media for 7 days. ALP and Alizarin Red S staining showed reduced ALP activity and mineralization upon rhLOXL2 treatment (Figs. $4 A$ and $4 B$ ). In accordance, ALP mRNA expression levels were decreased (Fig. $4 C$ ). The mRNA expression levels of DMP-1 and DSPP, odontoblast-selective genes, were also decreased during odontogenic differentiation (Figs. 4D and 4E). These data suggest that LOXL2 has a negative effect on hDPSCs differentiation into odontogenic/osteogenic cells.

\section{DISCUSSION}

In this study, we showed that lysyl oxidase-like 2 (LOXL2) expressions are decreased during odontogenic differentiation in hDPSCs. In addition, exogenous application of human recombinant LOXL2 protein resulted in a decrease of odontogenic differentiation. LOXL2 was initially identified as an over-expressed gene in senescent fibroblasts, and LOXL2 upregulation has often been reported in invasive metastatic tumors, compared to noninvasive tumors (Bignon et al., 2011). Furthermore, LOXL2 has been shown to mediate the induction of epithelialmesenchymal transition via repression of E-Cadherin expression, suggesting a functional role for LOXL2 in tumor progression. It seems that LOXL2 is involved in the maintenance of mesenchymal or fibroblastic characteristics. These evidences also indicate that LOXL2 may play roles to cell proliferation since both cancer cells and stem cells have characteristics of cell cycle progression. Thus, in accordance of our data, it is postulated that LOXL2 can be decreased upon losing cell proliferation characteristics and gaining cell differentiation stages.

It is of interest to note that the expression levels of LOXL2 are very low or undetectable in differentiating MC3T3-E1 osteoblasts (Atsawasuwan et al., 2005). The LOXL2 mRNA expression is also down-regulated in bone marrow stromal cells during differentiation (Monticone et al., 2004). In addition, it is also reported the expression of LOX isoforms including LOX, LOXL1, LOXL3, and LOXL4 were all upregulated by rhBMP-2 treatment. Of LOX isoforms, only LOXL2 expression was continuously diminished with the treatment (Kaku et al., 2007). Since, it is known that there are similarities between odontogenic differentiation and osteogenic differentiation, all these data support our findings that LOXL2 alone decreased during odontogenic differentiation of hDPSCs. Although the LOXL2 regulation is unique among LOXL family in odontogenic and osteogenic differentiation. It turns out so far that there is nothing yet unique in LOXL2 molecular structure. It would be meaningful if we could reveal any detailed special structure of LOXL2 in the future study.

Based on the conservation of the C-terminal domains, LOXL2 was predicted to function as an amine oxidase. However, the amine oxidase activity of LOXL2 has not yet been thoroughly studied. Since it is known that LOX has enzymatic activity of cross-linking Type I Collagen, and thus may promote the hard tissue (bone and dentin) calcification, it is postulated that the LOXL2 might inhibit the LOX lysine cross-linking activity. Therefore, the LOXL2 might be decreased during odontogenic and osteogenic differentiation. However, recent report showed that the LOXL2 proteins had significant amine oxidase activity toward Collagen and Elastin (Kim et al., 2011). But, the amine oxidase activity of LOXL2 was not significantly inhibited by a specific inhibitor of LOX, suggesting that LOX and LOXL2 may have different biochemical mechanisms for modulation of the amine oxidase activity (Kim et al., 2011). Our data showed that LOXL2, but not other LOX family members, was specifically decreased in hDPSCs when subjected to differentiation in mineralization media. All these evidences, to some extent, explain why the LOXL2 behave differently from other LOX families during odontogenic differentiation. Though the functions of LOXL2 in odontoblasts and osteoblasts are still not well understood, this particular form of LOXLs may be associated with certain odontogenic differentiation process and regulate hDPSCs differentiation process.

In conclusion, our results indicate that LOXL2 is negatively regulated during hDPSCs differentiation to mineralizing cells and rhLOXL2 suppressed the odontogenic differention. It is suggested that the inhibition of the LOXL2 might promote hDPSCs differentiation.

Note: Supplementary information is available on the Molecules and Cells website (www.molcells.org).

\section{ACKNOWLEDGMENTS}

This work was supported by a grant from the National Research Foundation (NRF) in Korea that was funded by the Ministry of Education, Science and Technology (No. 20110019355, 
20100026741, 2012М3А9С6049716, \& 20100028091) and Fellowship Foundation.

\section{REFERENCES}

Atsawasuwan, P., Mochida, Y., Parisuthiman, D., and Yamauchi, M. (2005). Expression of lysyl oxidase isoforms in MC3T3-E1 osteoblastic cells. Biochem. Biophys. Res. Commun. 327, 10421046.

Bignon, M., Pichol-Thievend, C., Hardouin, J., Malbouyres, M., Brechot, N., Nasciutti, L., Barret, A., Teillon, J., Guillon, E., Etienne, E., et al. (2011). Lysyl oxidase-like protein-2 regulates sprouting angiogenesis and type IV collagen assembly in the endothelial basement membrane. Blood 118, 3979-3989.

Chai, Y., and Slavkin, H.C. (2003). Prospects for tooth regeneration in the 21st century: a perspective. Microsc. Res. Tech. 60, 469479.

d'Aquino, R., Graziano, A., Sampaolesi, M., Laino, G., Pirozzi, G. De Rosa, A., and Papaccio, G. (2007). Human postnatal dental pulp cells co-differentiate into osteoblasts and endotheliocytes: a pivotal synergy leading to adult bone tissue formation. Cell Death Differ. 14, 1162-1171.

Duailibi, M.T., Duailibi, S.E., Young, C.S., Bartlett, J.D., Vacanti, J.P., and Yelick, P.C. (2004). Bioengineered teeth from cultured rat tooth bud cells. J. Dent. Res. 83, 523-528.

Gronthos, S., Mankani, M., Brahim, J., Robey, P.G., and Shi, S. (2000). Postnatal human dental pulp stem cells (DPSCs) in vitro and in vivo. Proc. Natl. Acad. Sci. USA 97, 13625-13630.

Hayashi, K., Cao, T., Passmore, H., Jourdan-Le Saux, C., Fogelgren, B., Khan, S., Hornstra, I., Kim, Y., Hayashi, M., and Csiszar, K. (2004a). Progressive hair loss and myocardial degeneration in rough coat mice: reduced lysyl oxidase-like (LOXL) in the skin and heart. J. Invest Dermatol. 123, 864-871.

Hayashi, K., Fong, K.S., Mercier, F., Boyd, C.D., Csiszar, K., and Hayashi, M. (2004b). Comparative immunocytochemical localization of lysyl oxidase (LOX) and the lysyl oxidase-like (LOXL) proteins: changes in the expression of LOXL during development and growth of mouse tissues. J. Mol. Histol. 35, 845-855.

Heo, S.H., Choi, Y.J., Lee, J.H., Lee, J.M., and Cho, J.Y. (2011). S100A2 level changes are related to human periodontitis. Mol. Cells 32, 445-450.

Jourdan-Le Saux, C., Tronecker, H., Bogic, L., Bryant-Greenwood, G.D., Boyd, C.D., and Csiszar, K. (1999). The LOXL2 gene encodes a new lysyl oxidase-like protein and is expressed at high levels in reproductive tissues. J. Biol. Chem. 274, 12939-12944.

Kagan, H.M., Reddy, V.B., Narasimhan, N., and Csiszar, K. (1995). Catalytic properties and structural components of lysyl oxidase. Ciba Found. Symp. 192, 100-115; discussion 115-121.
Kaku, M., Mochida, Y., Atsawasuwan, P., Parisuthiman, D., and Yamauchi, M. (2007). Post-translational modifications of collagen upon BMP-induced osteoblast differentiation. Biochem. Biophys. Res. Commun. 359, 463-468.

Kim, Y.M., Kim, E.C., and Kim, Y. (2011). The human lysyl oxidaselike 2 protein functions as an amine oxidase toward collagen and elastin. Mol. Biol. Rep. 38, 145-149.

Koyama, N., Okubo, Y., Nakao, K., and Bessho, K. (2009). Evaluation of pluripotency in human dental pulp cells. J. Oral Maxillofac. Surg. 67, 501-506.

Lee, E.H., Park, H.J., Jeong, J.H., Kim, Y.J., Cha, D.W., Kwon, D.K., Lee, S.H., and Cho, J.Y. (2010). The role of asporin in mineralization of human dental pulp stem cells. J. Cell. Physiol. 226, 1676-1682.

Maki, J.M., and Kivirikko, K.I. (2001). Cloning and characterization of a fourth human lysyl oxidase isoenzyme. Biochem. J. 355, 381-387.

Monticone, M., Liu, Y., Tonachini, L., Mastrogiacomo, M., Parodi, S., Quarto, R., Cancedda, R., and Castagnola, P. (2004). Gene expression profile of human bone marrow stromal cells determined by restriction fragment differential display analysis. J. Cell. Biochem. 92, 733-744.

Morsczeck, C., Schmalz, G., Reichert, T.E., Vollner, F., Galler, K., and Driemel, O. (2008). Somatic stem cells for regenerative dentistry. Clin. Oral Invest. 12, 113-118.

Narayanasamy, A., Ahn, J.M., Sung, H.J., Kong, D.H., Ha, K.S., Lee, S.Y., and Cho, J.Y. (2011). Fucosylated glycoproteomic approach to identify a complement component 9 associated with squamous cell lung cancer (SQLC). J. Proteomics 74, 2948- 2958.

Rodriguez, C., Martinez-Gonzalez, J., Raposo, B., Alcudia, J.F., Guadall, A., and Badimon, L. (2008). Regulation of lysyl oxidase in vascular cells: lysyl oxidase as a new player in cardiovascular diseases. Cardiovasc. Res. 79, 7-13.

Saito, H., Papaconstantinou, J., Sato, H., and Goldstein, S. (1997). Regulation of a novel gene encoding a lysyl oxidase-related protein in cellular adhesion and senescence. J. Biol. Chem. 272, 8157-8160.

Tretter, V., Altmann, F., and Marz, L. (1991). Peptide-N4-(N-acetylbeta-glucosaminyl)asparagine amidase $\mathrm{F}$ cannot release glycans with fucose attached alpha 1--3 to the asparagine-linked $\mathrm{N}$ acetylglucosamine residue. Eur. J. Biochem. 199, 647-652.

Yokose, S., Kadokura, H., Tajima, Y., Fujieda, K., Katayama, I., Matsuoka, T., and Katayama, T. (2000). Establishment and characterization of a culture system for enzymatically released rat dental pulp cells. Calcif. Tissue Int. 66, 139-144.

Yu, J., He, H., Tang, C., Zhang, G., Li, Y., Wang, R., Shi, J., and Jin, Y. (2010). Differentiation potential of STRO-1+ dental pulp stem cells changes during cell passaging. BMC Cell Biol. 11, 32 . 